This is an electronic reprint of the original article. This reprint may differ from the original in pagination and typographic detail.

Author(s): Harjupatana, Tero; Alaraudanjoki, Jarno; Kataja, Markku

Title: $\quad$ X-ray tomographic method for measuring three-dimensional deformation and water content distribution in swelling clays

Year: $\quad 2015$

Version:

Please cite the original version:

Harjupatana, T., Alaraudanjoki, J., \& Kataja, M. (2015). X-ray tomographic method for measuring three-dimensional deformation and water content distribution in swelling clays. Applied Clay Science, 114(September), 386-394.

https://doi.org/10.1016/j.clay.2015.06.016

All material supplied via JYX is protected by copyright and other intellectual property rights, and duplication or sale of all or part of any of the repository collections is not permitted, except that material may be duplicated by you for your research use or educational purposes in electronic or print form. You must obtain permission for any other use. Electronic or print copies may not be offered, whether for sale or otherwise to anyone who is not an authorised user. 


\title{
X-ray tomographic method for measuring three-dimensional deformation and water content distribution in swelling clays
}

\author{
Tero Harjupatana*, $^{*}$, Jarno Alaraudanjoki ${ }^{a}$, and Markku Kataja ${ }^{a}$ \\ aDepartment of Physics, University of Jyväskylä, P.O. Box 35 (YFL), \\ FI-40014, Finland
}

\begin{abstract}
A non-invasive method for simultaneous measurement of the 3D displacement field and the water content distribution of a wetted solid material is developed. The method is based on comparison of X-ray tomographic images of a material sample in the reference state and in the wetted and deformed state. The deformation and water content analyses were successfully compared with numerical results for a cylindrical rubber test sample under axial compression, and with gravimetric results from axially wetted and sliced cylindrical bentonite samples, respectively. The methods were applied in a $4 \mathrm{D}$ study (three spatial dimensions and time) of wetting and deformation of purified swelling bentonite doped with glass tracer particles, and wetted with synthetic groundwater. The results obtained for bentonite samples are repeatable and appear qualitatively correct and plausible. They are useful e.g. in validating models involving transport of water and the resulting swelling deformation of bentonite. The method is potentially applicable also in other processes involving liquid transport and deformation such as wetting/swelling and drying/shrinking of heterogeneous materials. A prerequisite for the applicability of the method is that the material contains sufficient amount of local inhomogeneities visible and identifiable in successive tomographic images to facilitate deformation analysis, and that change in water content affects the total density enough to be observable in X-ray images.
\end{abstract}

Keywords: X-ray tomography; Water content; Water transport; Deformation; Bentonite; Swelling

\section{Introduction}

Transport of liquids in partially saturated solid materials and the possible deformation of the material induced by local changes in liquid content are of great interest in many areas of

${ }^{*}$ Corresponding author.

E-mail: tero.t.harjupatana@jyu.fi 
materials science and technology. A number of complex physical and chemical mechanisms contribute to such transport and deformation in processes involving wetting or drying of e.g. soils, building materials, foods and various biological materials (Moldrup et al., 2001; Carmeliet and Roels, 2001; Saguy et al., 2005; Meinzer, 2002). Theoretical approaches based on first principles towards modeling these processes tend to become complicated, and phenomenological input is often required. Measuring the total liquid content and global deformation of a wetting/drying material sample is rather straightforward by conventional gravimetric and morphological methods (Gardner et al., 2000; Orteu, 2009). In their early work, Anderson et al. used medical X-ray tomographic device for rapid non-destructive measurement of bulk density and water content of soil samples (Anderson et al., 1988). At least rough local information can be obtained by destructive segmenting of the sample. Non-invasive techniques based e.g. on nuclear magnetic resonance, electric properties of material, and various modalities of tomography have also been used for measuring the local three-dimensional liquid content distribution (Herrmann et al., 2002; Aregawi et al., 2013; Mukhlisin et al., 2012; Huisman et al., 2003) or the local deformation of material samples in various mechanical conditions (Peth et al., 2010; Bart-Smith et al., 1998). Very few efforts appears to have been made towards simultaneous non-destructive measurement of the evolution of both the liquid content and the local deformation field of a material sample during wetting or drying process. Availability of such a measurement method would be potentially very useful for experimental research of processes involving liquid transport and the resulting deformation, and for development and validation of theoretical models of such processes. In this work, we introduce a method based on X-ray microtomography for non-destructive simultaneous measurement of three-dimensional distribution of local water content and displacement field of a wetted material. The method is applied in monitoring the swelling behavior of a wetting bentonite sample.

With X-ray tomography, the spatial distribution of the linear X-ray attenuation coefficient in the sample is obtained (Stock, 2008). The data is conveniently represented as a threedimensional grayscale image allowing not only visualization but also quantitative study of the internal structure of many heterogeneous materials. The different material components of a multiphase material can be directly observed provided that the typical size scale of the phase domains is larger than the imaging resolution, that the difference between the values of the attenuation coefficient of various phases is large enough, and that the phase configuration remains stable during the time of tomographic imaging. In such a case, the relative amount and distribution of various phases can be found in a straightforward manner using 3D image analysis techniques (Wildenschild et al., 2005; Tippkötter et al., 2009; Riedel et al., 2012). Sammartino et al. have used such an approach together with a relatively fast medical X-ray tomographic scanner in order to characterize flow in macropores of soil samples (Sammartino et al., 2012).

However, although we consider here a solid material partially saturated with a water, i.e. a three-phase system of solid, water and gas, we can not assume phase separation in the size scale given by the resolution of the X-ray tomographic technique available $(\sim 1 \mu \mathrm{m})$. Instead, each material volume of the size scale given by the imaging resolution, i.e. the image voxel, can contain all three phases that contribute to the total value of the attenuation constant and thus of the grayscale value of the voxel. A single tomographic image of a material sample can thus not provide direct information on the relative abundances of different phases. Such 
a case was also considered (Sammartino et al., 2012) in evaluating the amount of water contained in soil matrix regions with small-scale porosity in the vicinity of macropores.

In what follows, we neglect the effect of gas phase, and confine ourselves to cases where the attenuation coefficients of the bulk solid and water are the same order of magnitude such that the changes of water content in the solid material are observable with X-ray tomography. We also expect, that the water transport in the material is slow enough such that the phase configuration can be considered approximately stationary during tomographic imaging. (Depending on the technique used, the typical time required for a single X-ray tomographic scan can vary from a few minutes to several days.) Furthermore, we assume that an experimental correlation formula for the dependence of X-ray attenuation coefficient on solid and water contents can be found using some independent calibration method such as gravimetric measurement. In the case that the solid phase content is known, the water content distribution in the partially saturated state may then be found in a straightforward manner by utilizing the X-ray tomographic image. In many practical cases however, change in water content induces considerable solid phase deformation. Then, the local solid content in the sample is not known making it impossible to utilize the calibration data and find the water content based on the measured total attenuation coefficient only. Solution to the problem can be sought provided that an X-ray tomographic image of the same physical sample is taken in a reference state of known solid density. Using image correlation techniques on the images of the reference state and the partially saturated state of interest, called the 'current state' in what follows, the three-dimensional displacement field of the solid phase may be found. Given the reference state solid density, this deformation information can be used to calculate the local solid density distribution in the current state. Together with the total attenuation coefficient data (the X-ray tomographic image), the calibration correlation thus yields the water density distribution in the current state. A prerequisite for successful analysis of the displacement field is that the material contains local structures visible in tomographic images of both the reference state and the current state. In some cases, this may be achieved by doping the material with suitable marker particles.

The primary motivation for the present work has been the need for developing efficient experimental methods for studying groundwater transport and swelling mechanisms of bentonite. This type of clay can absorb large amount of water, swell multiple times of its original volume, and produce large swelling pressure if wetted confined in a closed space. Fully saturated compacted bentonite is considered as effective barrier for transport of water and various chemicals. Due to its unique properties, bentonite is widely used in many applications of soil mechanics as a buffer and sealing material. It is also planned to be used as buffer material in some repository concepts for used nuclear fuel. The long-term purpose of this work is to utilize the X-ray tomographic techniques in a '4D imaging' sense, i.e. monitoring the evolution of water content and deformation of wetting and swelling bentonite samples in three dimensions and as a function of time, thereby producing detailed experimental data for supporting development and validation of hydromechanical models of bentonite. 


\section{Methods}

\subsection{X-ray tomography}

$\mathrm{X}$-ray imaging is based on attenuation of X-rays in a material. The intensity $I$ of a narrow monochromatic X-ray beam is attenuated in a material according to the Beer-Lambert law (Hubbell and Seltzer, 1996)

$$
I=I_{0} \cdot \mathrm{e}^{-\int \mu(x) d x}
$$

where $\mu$ is the linear attenuation coefficient (LAC) which can depend on position $x$ along the beam path. The detector of a typical X-ray tomographic device consists of a phosphorescent screen that converts X-rays to visible light, and a digital camera. A single X-ray projection image of a sample represents the intensity ratio $\left(I / I_{0}\right)$ detected on such a two-dimensional detector. In a typical X-ray tomographic imaging procedure, of the order of one thousand $\mathrm{X}$-ray projection images of the sample are taken from different directions by rotating the

sample in the X-ray beam. The three-dimensional distribution of LAC is then reconstructed from the projection images by a computer. The reconstructed data is represented as a threedimensional image (stack of two-dimensional cross-sectional images) of the sample. The grayscale 'voxel' values in such an image are linearly correlated with the actual LAC value in the sample.

It can be shown that for a given substance the LAC is proportional to its bulk density (Hubbell and Seltzer, 1996). The mass attenuation coefficient, defined as $\mu_{m}=(\mu / \rho)$, is thus independent of density but depends on X-ray energy and the atomic number of the substance. For compound material of several substances the LAC is given by

$$
\mu=\sum_{i}\left(\mu_{m, i} \cdot \rho_{i}\right)
$$

where $\mu_{m, i}$ and $\rho_{i}$ are the mass attenuation coefficient and the partial density of substance $i$, respectively (Hubbell and Seltzer, 1996). The partial density is defined as $\rho_{i}=\phi_{i} \tilde{\rho}_{i}$, where $\phi_{i}$ and $\tilde{\rho}_{i}$ are the volume fraction and the intrinsic material density of the substance, respectively (Soo, 1990).

The radiation source used in current laboratory scale tomographic devices is X-ray tube which produces a polychromatic X-ray beam. Since the attenuation constant depends on energy, the simple Beer-Lambert law, Eq. (1), does not exactly hold. However, in simple reconstruction algorithms, the effects of the wide X-ray energy spectrum are neglected leading to imaging artifacts such as beam hardening (Stock, 2008). Since the attenuation coefficient typically decreases with increasing energy, beam hardening appears in tomographic images as edges of the sample showing virtually more absorbing (denser) than the interior even for a homogeneous sample. For the present technique, beam hardening poses a challenge since it weakens the linear correlation between the grayscale value and the actual attenuation coefficient on which the method is heavily based on. The effects of beam hardening can be reduced by using metallic filters to cut the low energy part of the spectrum. A potential disadvantage of the filtering technique is that the overall intensity of the beam is decreased leading to longer imaging times. The remaining effects of the beam hardening effect can be corrected for in the reconstruction stage (Zou et al., 2011), whereby the approximate linear dependence 
of the total absorption coefficient and thus of the grayscale value on the partial densities can be retained. Another imaging defects typical to X-ray tomographic techniques include the ring artifact, which can be caused e.g. by a single faulty pixel of the detector camera, and the cone beam artifact related to approximations used in reconstruction algorithms for conical X-ray beam geometries (Valton et al., 2006; Hsieh et al., 2013). The effects of these artifacts can be reduced, if not removed, by specific correction algorithms included in the standard reconstruction software or by post-processing of reconstructed images. (Stock, 2008; Barrett and Keat, 2004)

The X-ray microtomographic device used in the present work was SkyScan 1172 desktop scanner which has a microfocus X-ray tube with maximum operating voltage of $100 \mathrm{kV}$ and maximum power of $10 \mathrm{~W}$. The full image size of the CCD camera is $4000 \times 2096$ pixels and the minimum pixel size is $0.7 \mu \mathrm{m}$. The best resolution (detail detectability) is approximately $5 \mu \mathrm{m}$. The maximum field-of-view size is $34 \mathrm{~mm} \times 18 \mathrm{~mm}$, achieved with $8.5 \mu \mathrm{m}$ pixel size setting. For the purposes of the present study the device was used in a reduced resolution mode with image size $1000 \times 524$ pixels of size $24 \mu \mathrm{m}$. These settings allow $24 \mathrm{~mm} \times 12.6$ $\mathrm{mm}$ field of view, and a relatively short imaging time.

\subsection{Experimental set-up and samples}

The samples used in the experiments were made of a commercial purified bentonite supplied in a powder form (MP Biomedicals Bentonite). The initial water content of the bentonite powder was measured gravimetrically using oven drying at $105^{\circ} \mathrm{C}$ and typical water content was around $7 \%$ by weight (this depends on storage conditions). In order to facilitate deformation measurement, hollow glass microspheres of diameter $\sim 100 \mu \mathrm{m}$ were mixed with the bentonite powder to act as inert tracer particles in the otherwise quite homogeneous material. Cylindrical samples of diameter $17 \mathrm{~mm}$ and height $10 \mathrm{~mm}$ were then made by compacting a weighed amount of bentonite powder in a mould into a predetermined mean solid phase partial density ('dry density') $\rho_{s 0}$ of 1.2 or $1.5 \mathrm{~g} / \mathrm{cm}^{3}$. The compacted bentonite sample was then carefully placed and confined in the sample holder (see Fig. 1), slightly pre-compressed to achieve firm contact with the inner surfaces of the sample casing.

During the experiment, the sample was held in approximately constant volume in a plastic (PEEK) tube and between cylindrical end-pieces. The end-pieces include wetting and venting channels, and glass sintered plates that allow water flow in the sample through the lower end surface, and escape of air through the upper surface. The wetting channel includes the necessary miniature valves and an air bleeding channel to allow for controlling the inflow of water in the sample and for removal of air from the channel and the lower sintered plate. Two versions of sample holder with sintered plate diameters $6 \mathrm{~mm}$ and $15 \mathrm{~mm}$ were used in the experiments. The sample holder equipped with the smaller sintered plate size was designed to produce a three-dimensional cylindrically symmetric set-up, while the sample holder with the larger sintered plate gives approximately one-dimensional axial wetting and swelling behavior. In practice, these symmetries may be violated to some extent due to inhomogeneities present in the samples. The sample holders also include force sensors for measuring swelling pressure at the top surface of the sample. The water used in wetting the bentonite sample was synthetic groundwater with total salt content of $0.26 \mathrm{~g} / \mathrm{l}$ (Allard pH 7 water (Huitti et al., 1998)). 


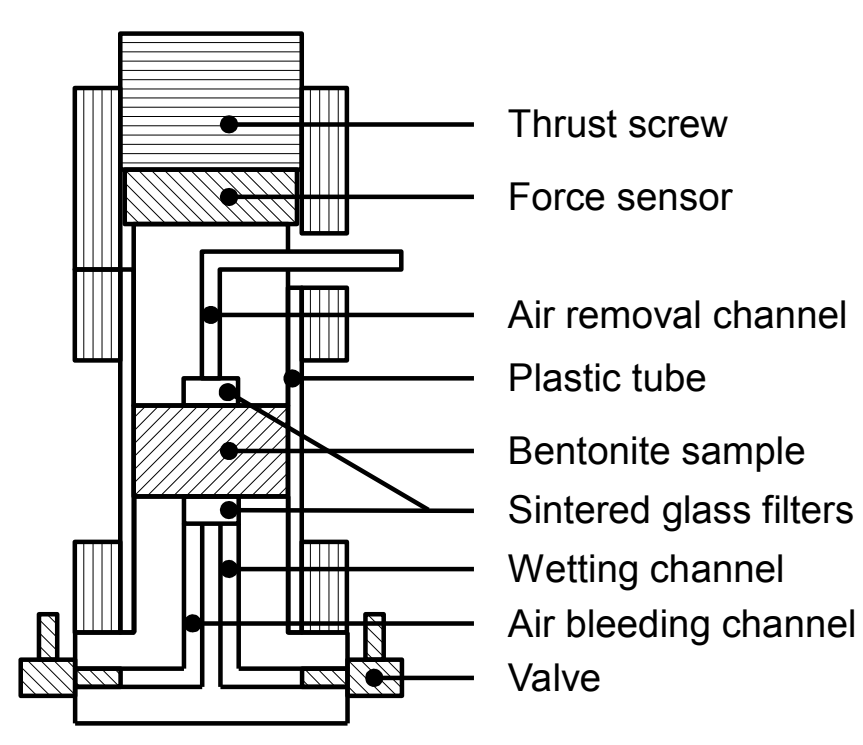

(a)

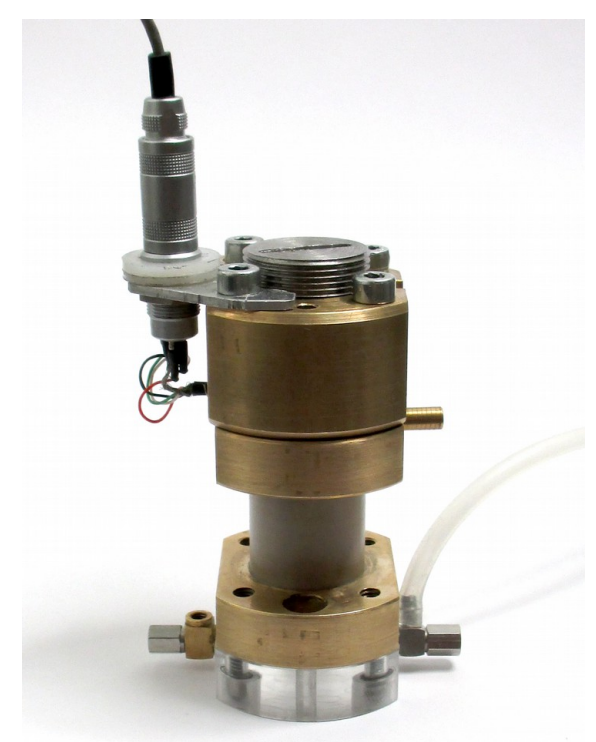

(b)

Figure 1: Schematic illustration (a) and a photographic image (b) of the sample holder.

The experiment was started by taking a reference state tomographic image of the nonwetted sample. After that, the wetting channels and the lower sintered plate were carefully filled with water and the whole sample holder was immediately weighed with hoses and wires disconnected. The sample holder was then connected to water supply and the wetting of the sample was initiated. The wetting was periodically interrupted and the sample holder with the partially saturated sample weighed, scanned in the tomographic device, weighed again and reconnected to water supply to resume wetting. The scanning time was about $45 \mathrm{~min}$, and the total time required for each scanning-weighing interval was about an hour. The procedure was repeated typically 10 times until the sample was completely saturated in about 1-2 weeks total time. The total water mass data obtained by weighings was used in calibrating the method for water content measurement, see Sect. 2.4. Examples of the X-ray projection image and the tomographic image of a bentonite sample used in this study are shown in Fig. 2.

\subsection{Deformation analysis}

The local displacement of the solid phase caused by swelling can, in principle, be found by comparing the tomographic images of the reference state and each of the partially wetted states of the sample, provided that both images contain enough tractable details. To facilitate such analysis with the present quite homogeneous purified bentonite material, the samples were doped with small amount of tracer particles, as discussed above. In addition to displacement due to swelling, intake of water in the sample causes changes in the local $\mathrm{X}$-ray attenuation coefficient and thus in grayscale values in the tomographic image of wetted states. While this phenomenon allows estimation of water content (see below) it has an unwanted side effect of hampering the analysis of local displacements by image correlation 


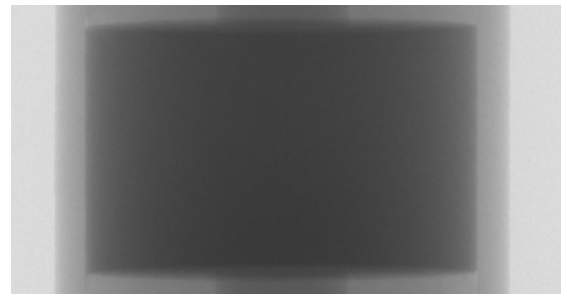

(a)

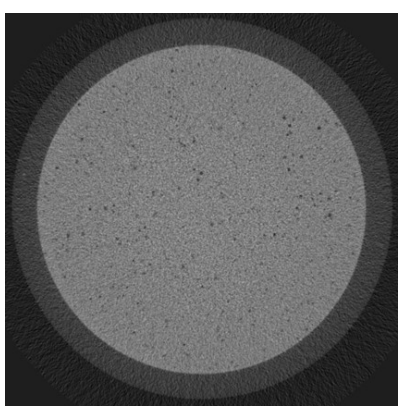

(b)

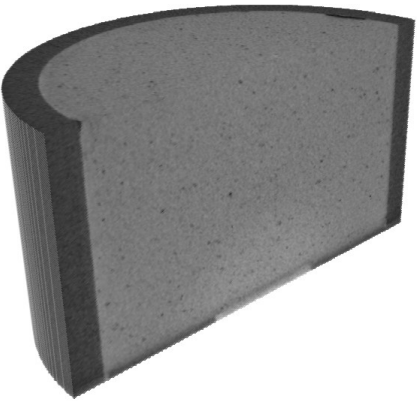

(c)

Figure 2: Single X-ray projection image (a), reconstructed horizontal slice (b) and three-dimensional volume image (half a sample) (c) of a cylindrical bentonite sample. The reconstructed images also show a section of the plastic sample holder tube.

techniques. Further improvement can be achieved by normalizing each tomographic image prior to displacement analysis by dividing it, voxel by voxel, by a suitably 3D mean filtered version of the same original image. The effect of this procedure to image quality is illustrated in Fig. 3.

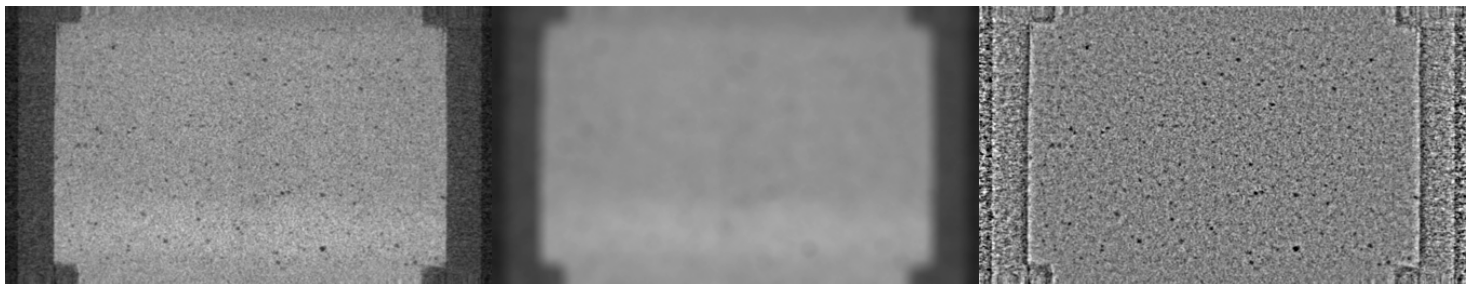

(a)

(b)

(c)

Figure 3: Normalization of a tomographic image for displacement analysis. The original image (a), the same image blurred by 3D mean filtering (b) and the normalized image, i.e. the original image divided by the filtered image (c).

The displacement vectors are determined in a three-dimensional grid defined in the reference state image. At every point of that grid, a control subimage, extracted from the normalized reference state image, is shifted voxel by voxel in the vicinity of the grid point and subtracted from its counterpart subimage in a normalized wetted state image. For each shift, the value of a penalty function defined as the sum of squares of the difference is calculated. The shift corresponding to the absolute minimum of the penalty function gives the first estimate for the displacement vector with a precision of one grid unit (voxel). A refined estimate for the displacement is then determined by fitting a second order polynomial function to the values of the penalty function at the location of penalty function minimum and its 26 nearest neighbors. The final displacement vector is then defined as the difference between position of the absolute minimum of this polynomial function and the original grid point. This refined block matching algorithm appears efficient and adequate for the present case where the deformation is relatively small and the added tracer particles facilitate image 
correlation. Application to other materials and deformation states may require using more advanced 3D image correlation algorithms for displacement analysis.

Given the measured displacement vector field $\vec{u}_{s}=\vec{u}_{s}(\vec{r})$, the experimental relative partial density change of the solid phase material point between the reference state and the deformed state with finite deformations is given by

$$
\Delta \rho_{s}(\vec{r})=-\rho_{s 0}(\vec{r}) \frac{\delta(\vec{r})}{1+\delta(\vec{r})},
$$

where $\rho_{s 0}$ is the solid phase partial density in the reference state, $\delta=\nabla \cdot \vec{u}_{s}$ is the relative volume change and $\vec{r}$ is the location of the material point in the reference state.

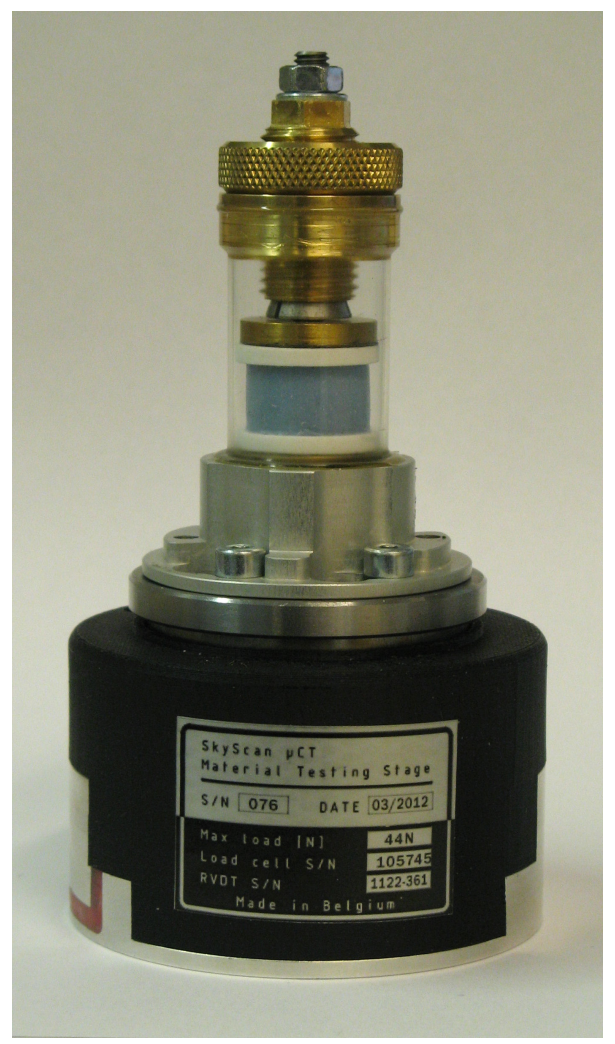

Figure 4: Sample holder (SkyScan $\mu C T$ Material Testing Stage) loaded with a rubber test sample used in validating the displacement analysis algorithm.

In order to test the deformation analysis algorithm, a cylindrical sample was made of twocomponent liquid rubber material doped with glass tracer particles similarly to the bentonite samples. Also, the shape and size of the rubber test sample were the same as the actual bentonite samples used in experiments. The rubber sample was placed in a material testing stage that allows tomographic imaging of the material under compression or tension, and is equipped with displacement and load sensors (Fig. 4). The cylindrical rubber sample was attached between the piston and the bottom plate of the testing stage so as to prevent lateral spread of the end surfaces. Otherwise, the test condition was unconfined. A reference tomographic image of the sample was taken at zero load. The sample was then compressed axially to total linear strain $\varepsilon \approx-0.035$ thereby inducing deformation into a barrel-like shape, and 


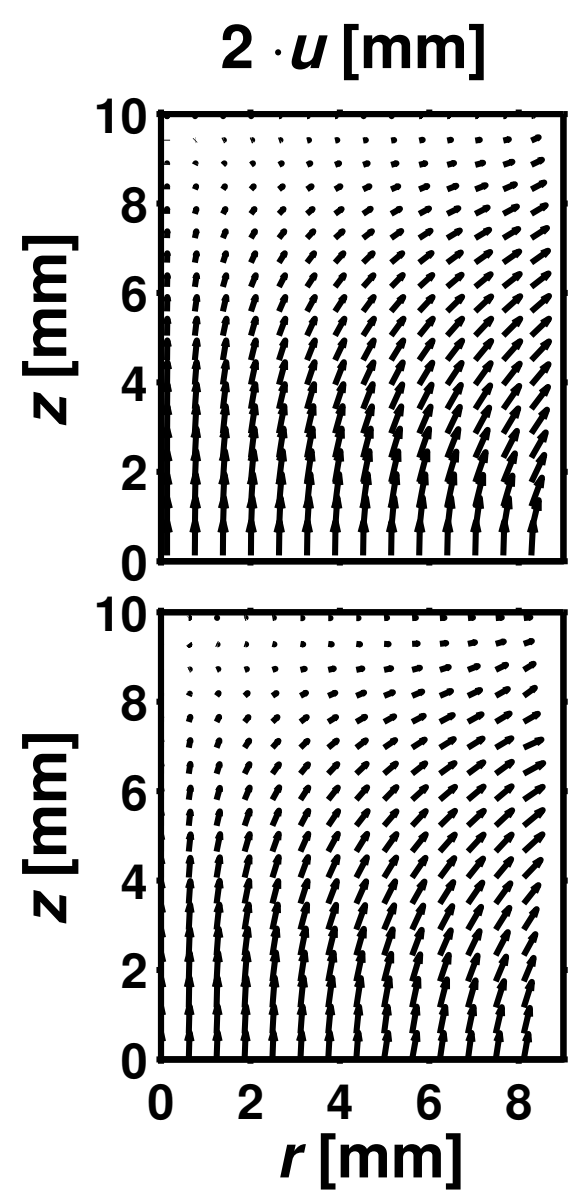

$u_{r}[\mathrm{~mm}]$
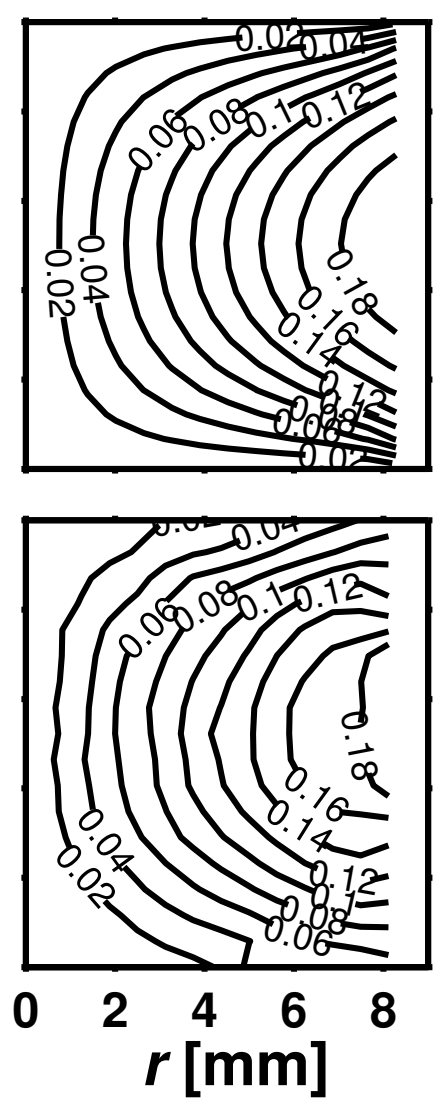

$u_{z}[\mathrm{~mm}]$
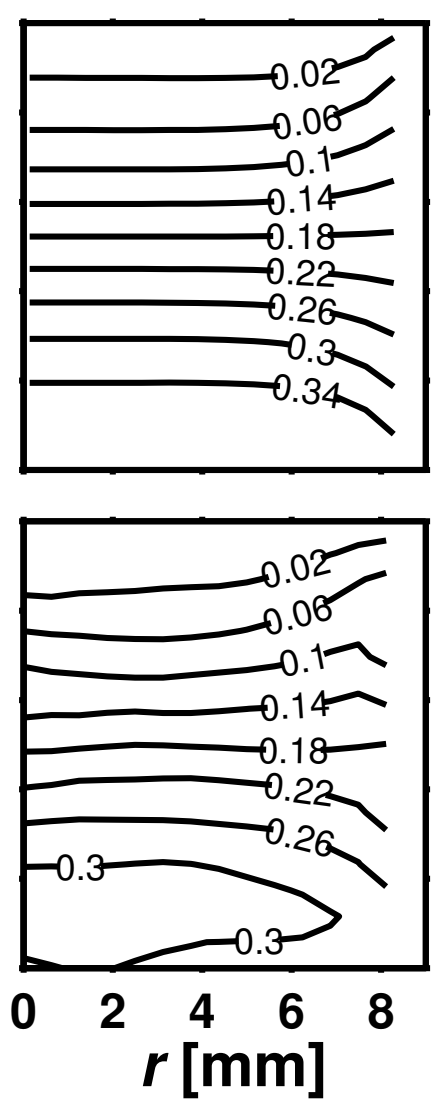

Figure 5: Numerical solution (COMSOL) for cylindrically symmetric displacement vector field and its radial and axial components of the axially compressed rubber test sample (upper images). The azimuthally averaged experimental result obtained by the refined block matching algorithm from tomographic images of the sample (lower images).

imaged again in this configuration. The refined block matching algorithm was used to calculate the displacement field between the unloaded reference state and the deformed state. The results, averaged over the azimuthal angle, were compared with a cylindrically symmetric numerical solution for the same set-up obtained by COMSOL software and treating rubber as a linearly elastic material. (A semi-analytical scaling solution for this problem also exists (Watanabe, 1996), but is rather complicated and still involves numerical solution of a differential equation.) It turns out that the only material parameter affecting the shape of the sample in this set-up is Poisson's ratio. Rubber materials are usually considered nearly incompressible with Poisson's ratio close to $\nu \approx 0.5$. Here, the value $\nu=0.49$ was used for computational reasons. The experimental and numerical displacement fields of the rubber test sample are shown in Fig. 5 indicating very close qualitative and quantitative agreement. 


\subsection{Water content distribution}

As discussed above, an X-ray tomographic image of a heterogeneous material represents the three-dimensional spatial distribution of the linear attenuation coefficient $\mu$ in a sample such that the grayscale value $G(\vec{r})$ of a voxel in a given location $\vec{r}$ of the image depends approximately linearly on the LAC value $\mu(\vec{r})$. According to Eq. (2) the grayscale value of each voxel of a tomographic image of partially saturated bentonite sample is thus linearly correlated with the partial densities of solid, water and air in the corresponding location of the sample. In the present case, the effect of presence of air on total attenuation constant can be considered small, and will be neglected. We thus adopt a linear relation between the grayscale value $G$ and the partial densities $\rho_{s}$ and $\rho_{w}$ of solid and water phases, respectively, i.e.

$$
G=C+\alpha_{s} \cdot \rho_{s}+\alpha_{w} \cdot \rho_{w},
$$

where $C, \alpha_{s}$ and $\alpha_{w}$ are coefficients that may depend on energy spectrum, material composition and various imaging, reconstruction and image post-processing parameters.

The unknown coefficients in Eq. (4) were estimated by gravimetric measurement for an extensive set of calibration samples at varying solid and water content. Of the order of 100 calibration data points were thus collected, a subset of which is shown in Fig. 6. The measured relationship between the partial densities and the grayscale value indeed appears approximately linear in the density range relevant for the present experiment. The fitted values and error limits for coefficients appearing in Eq. (4) are

$$
\begin{aligned}
C & =18.9 \pm 3.8 \\
\alpha_{s} & =78.5 \pm 2.7 \mathrm{~cm}^{3} / \mathrm{g} \\
\alpha_{w} & =42.8 \pm 2.4 \mathrm{~cm}^{3} / \mathrm{g} .
\end{aligned}
$$

Notice that these values are valid only for the tomographic imaging and reconstruction parameter values used in calibration, and the same parameters must be used in actual measurements. Also shown in Fig. 6 are the grayscale values obtained for pure air and for the plastic sample holder tube material (PEEK) found in each tomographic image. This is to monitor and verify stability of the tomographic device during the long imaging period.

Given the three-dimensional tomographic images $G$ and $G_{0}$ of the current state and the reference state, respectively, the difference image between the two states is given by

$$
\Delta G=G_{P}-G_{0}=\alpha_{s} \cdot \Delta \rho_{s}+\alpha_{w} \cdot \Delta \rho_{w} .
$$

Here, $G_{P}$ is the pulled-back image of current state created by interpolating image $G$ such that $G_{P}(\vec{r})=G\left(\vec{r}+\vec{u}_{s}(\vec{r})\right)$, where $\vec{u}_{s}(\vec{r})$ is the displacement of a material point located at $\vec{r}$ in the reference state (see Sect. 2.3). The change of partial density of the water is thus given by

$$
\Delta \rho_{w}=\frac{\Delta G-\alpha_{s} \cdot \Delta \rho_{s}}{\alpha_{w}}
$$


where the change of the solid phase density $\Delta \rho_{s}$ is given by Eq. (3) for the measured current state displacement field. Finally, the water content in the current state is obtained as

$$
\eta=\frac{\rho_{w}}{\rho_{s}}=\frac{\rho_{w 0}+\Delta \rho_{w}}{\rho_{s 0}+\Delta \rho_{s}},
$$

where the reference state density of solid and water phases is given by

$$
\begin{aligned}
\rho_{s 0} & =\frac{G_{0}-C}{\alpha_{s}+\eta_{0} \alpha_{w}} \\
\rho_{w 0} & =\eta_{0} \rho_{s 0} .
\end{aligned}
$$

Here, $\eta_{0}$ is the reference state water content, assumed to be constant.

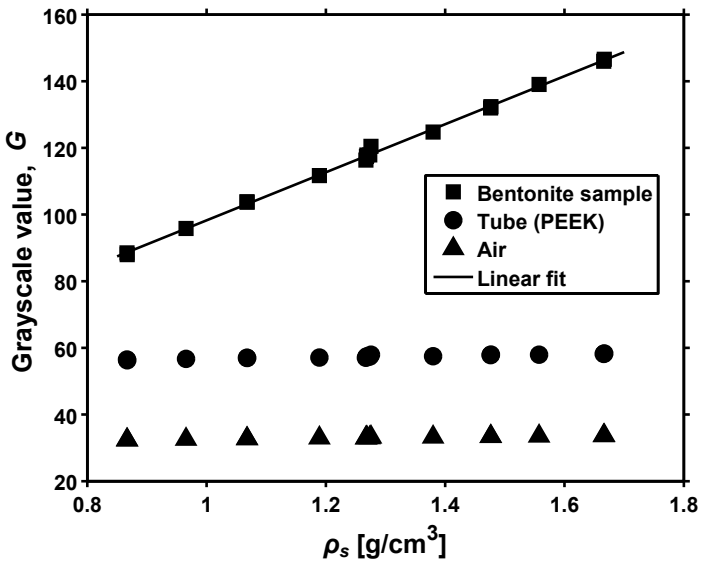

(a)

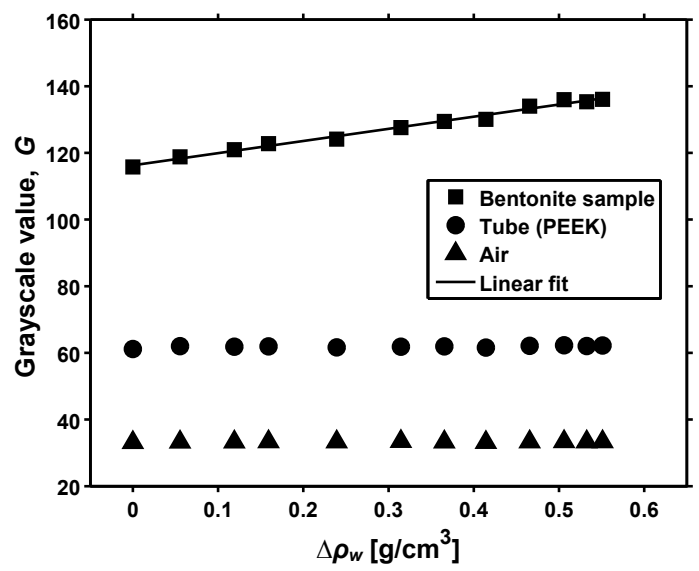

(b)

Figure 6: (a) Average grayscale value of a bentonite sample as a function of average dry density of the solid phase. Each data point corresponds to a different sample made of bentonite powder (with constant initial water content) by compacting it to a desired average solid phase density. (b) Average grayscale value of the sample as a function of average partial density change of water. This data is from a single sample during wetting from initial to fully saturated state. The constant values of the grayscale values of air and PEEK plastic, included in the figures for reference, are indicative of the tomographic device stability between scans.

The tomographic water content analysis method discussed above was compared with results from a straightforward gravimetric analysis of subsamples obtained by slicing a partially wetted test sample. To this end, a dry bentonite sample was first placed in a sample holder with $15 \mathrm{~mm}$ sintered plates (see Sect. 2.2), weighed and imaged to obtain reference state data. The sample was then wetted for $22 \mathrm{~h}$ so as to develop an axial water content distribution. After the wetting phase, the sample was again weighed and scanned according to the procedures discussed above. Immediately after the latter scan, the sample was carefully cut horizontally into 10 slices of thickness about $1 \mathrm{~mm}$. The water content of each slice was determined gravimetrically using oven drying at $105^{\circ} \mathrm{C}$. The results obtained from the 


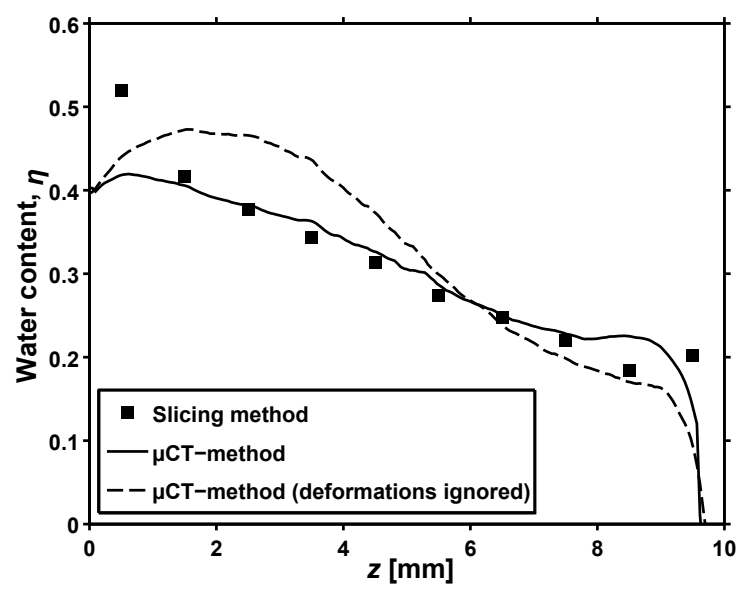

Figure 7: A comparison of 1D water content distribution measured gravimetrically from sliced bentonite sample and by the tomographic difference image method (Eq. 8). The latter result is shown including and ignoring the effects of local deformations due to swelling.

gravimetric measurement and from the tomographic imaging method are shown in Fig. 7 indicating reasonably good correspondence between the two results in regions well inside the sample. The deviation between the results near the lower and upper end of the test sample most likely arises due to the cone beam reconstruction artifact which tends to mix LAC values between the sample and its surroundings in the vicinity of the horizontal end surfaces of the sample (Valton et al., 2006; Hsieh et al., 2013). Also shown in Fig. 7 is the tomographic analysis result obtained by neglecting the effects of deformation (i.e. assuming $\vec{u}_{s}=0$ ). This result clearly shows the importance of the simultaneous deformation analysis in estimating the water distribution for the present strongly swelling clay material.

\section{Results}

The X-ray tomographic imaging and image analysis methods described above yield threedimensional displacement field and water content distributions in the material sample at selected times during the slow wetting process. Typical examples of such results for a bentonite sample wetted in the sample holder chamber with $6 \mathrm{~mm}$ sintered plate are visualized in Fig. 8. Figure 9 shows similar results, but averaged over the azimuthal angle for two experiments with sample holders with $6 \mathrm{~mm}$ and $15 \mathrm{~mm}$ sintered plates, corresponding to three-dimensional cylindrically symmetric and approximately one-dimensional axial set-up, respectively. Displacement and water content profiles averaged further over the radial direction for the set-up with $15 \mathrm{~mm}$ sintered plate are shown in Fig. 10. Finally, the swelling stress measured at the non-wetted end of the samples is shown in Fig. 11. In all results presented here, the average dry density of the samples was $1.2 \mathrm{~g} / \mathrm{cm}^{3}$.

Altogether, the results on displacement distributions obtained by the X-ray tomographic analysis appear consistent and repeatable in the entire sample region. The most important source of error in the deformation analysis is the spurious displacement vector values that 

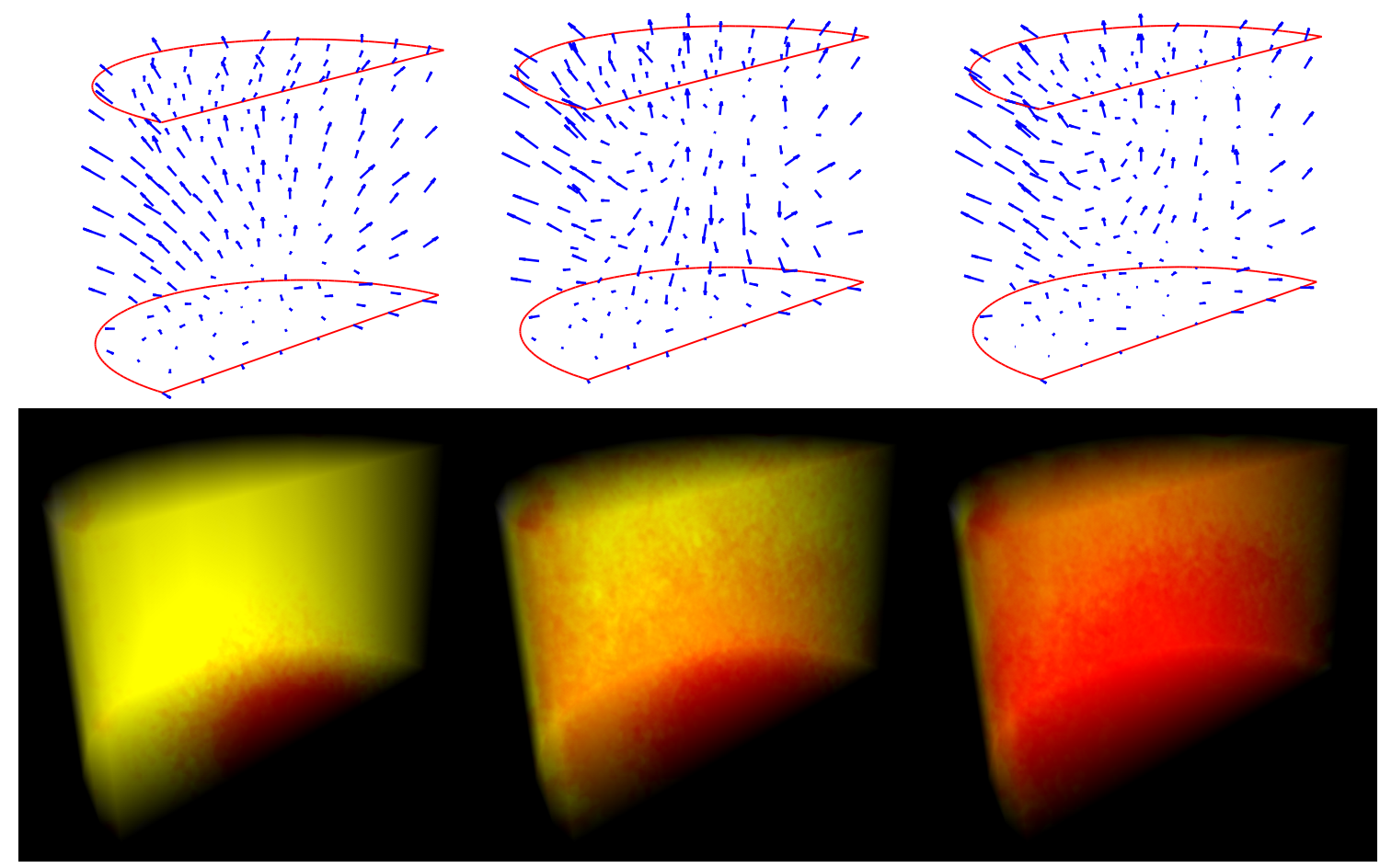

Figure 8: Three-dimensional visualization of displacement field (upper images) and water content distributions (lower images) at wetting times of $7 \mathrm{~h}, 25 \mathrm{~h}$ and $71 \mathrm{~h}$, for half a sample (see Fig. 2 (c)) in a sample holder with $6 \mathrm{~mm}$ sintered plate. The displacement vectors are shown scaled by a factor of 10 . The color code for water content is qualitatively same as in Fig. 9.

occasionally appear as a result of false local minima found by the image correlation algorithm. Such values may contribute to fluctuations visible in the three-dimensional displacement vector field in Fig. 8, but do not seem to have significant contribution to azimuthally averaged results shown in Fig. 9. Notice however, that part of the fluctuation visible in the threedimensional case may be due to actual inhomogeneity of the solid phase density and water content in the sample. The measured water content distributions and their evolution during the wetting time also appear highly plausible, in general. Near the sample boundaries the results appear impaired, to some extent, e.g. due to reconstruction artifacts arising from conical X-ray beam geometry and from the presence of surfaces with large density gradient (see also Fig. 7). At the beginning of the experiment, the water transport is relatively fast and this may produce some blurring to the images due to the relatively long imaging time characteristic of the used tomographic scanner. Although not necessarily significant from the point of view of the quality of individual images, these effects can decrease the accuracy of the water content analysis based on small differences in the grayscale value of the reference and current images.

Another experimental issue affecting the accuracy of water content measurement is the incomplete stability of the X-ray source and detector. For accurate results, very good stability is required during each individual scan and between the scans during the experiment, lasting typically up to 2 weeks in the present case. The effects of the intensity variations observed in 

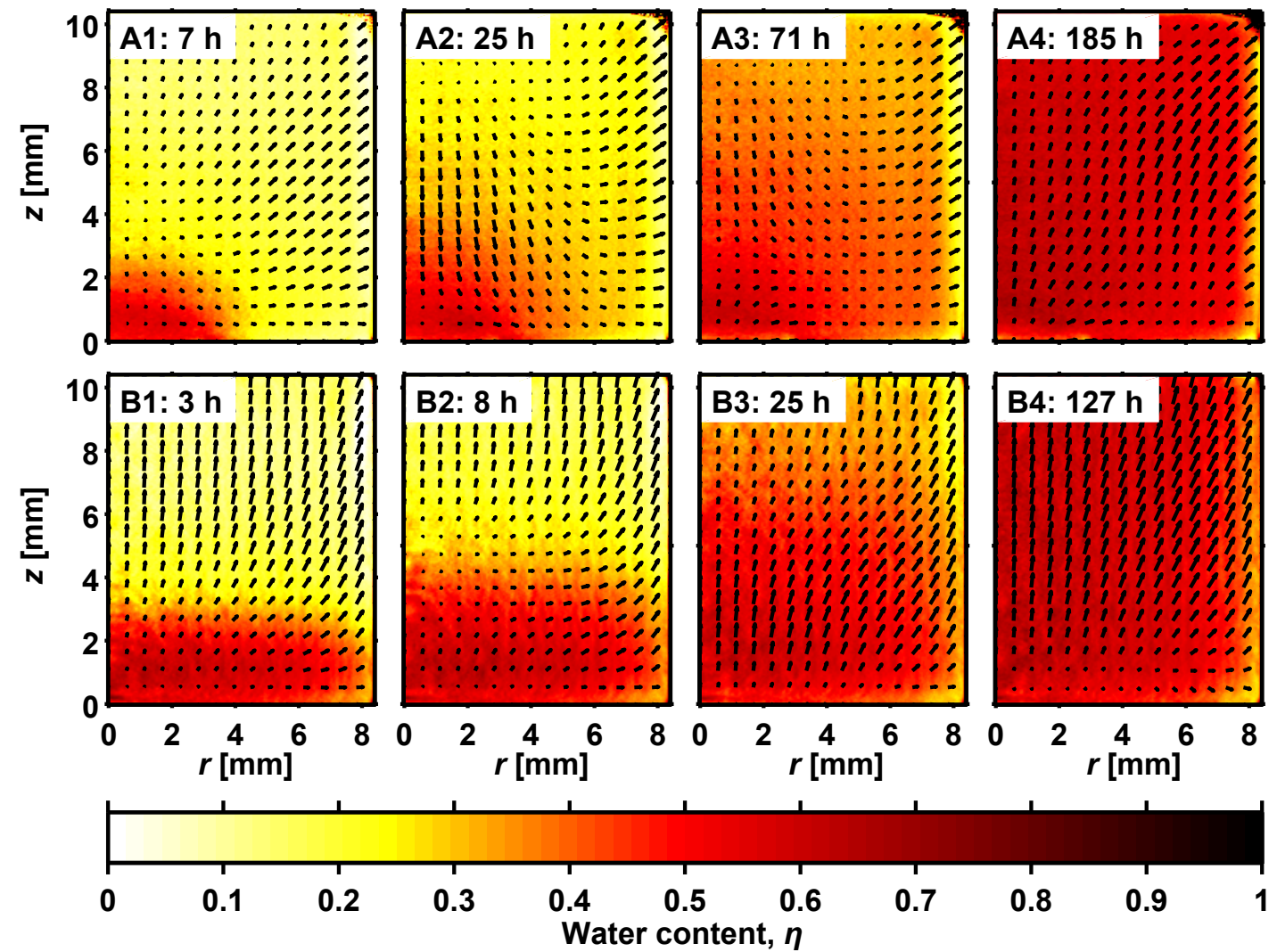

Figure 9: Water content and displacement vector fields averaged azimuthally for two different wetting geometries with $6 \mathrm{~mm}$ and $15 \mathrm{~mm}$ diameter sintered plates, labeled as A and B, respectively. The displacement vectors are drawn scaled by a factor of 5 . The wetting times are indicated in the image labels.

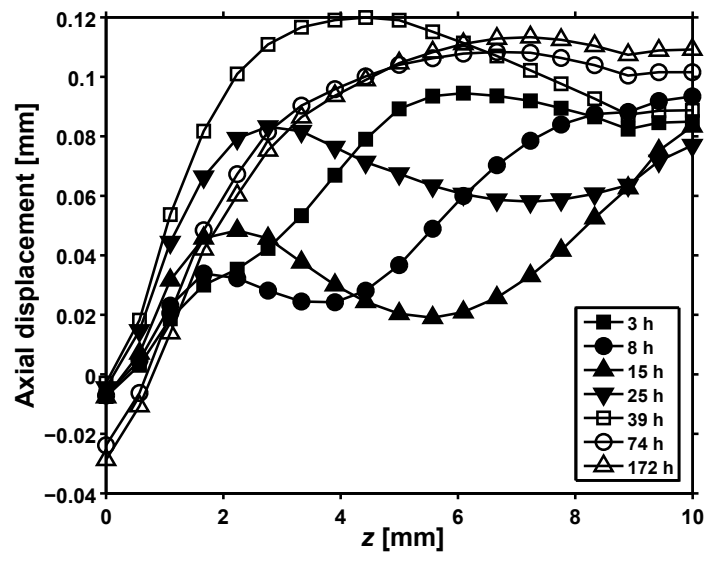

(a)

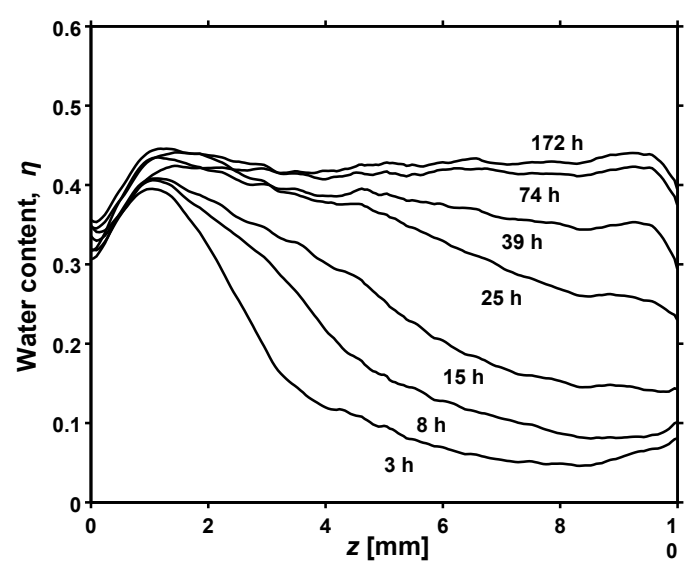

(b)

Figure 10: Evolution of the averaged axial displacement (a) and water content profiles (b) for a bentonite sample wetted in the sample holder with $15 \mathrm{~mm}$ sintered plate. 


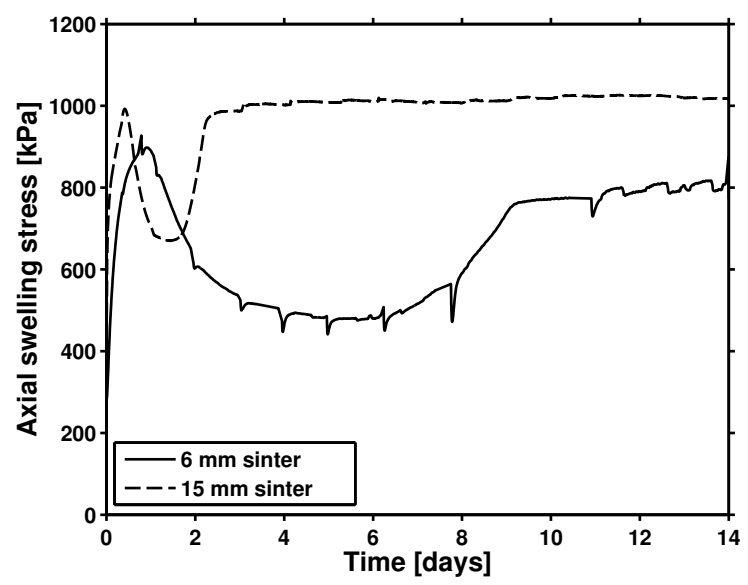

Figure 11: Axial swelling stress measured from non-wetted end of the sample holder as a function of wetting time for two bentonite samples wetted through $6 \mathrm{~mm}$ and $15 \mathrm{~mm}$ sintered plates. The notches visible especially in the curve corresponding to the $6 \mathrm{~mm}$ sintered plate arise due to repeated short interruptions of wetting for X-ray tomographic imaging.

practice were partially corrected by the standard flat field corrections performed separately for each scan. However, the minor temporal instability inherent in the device does contribute to the error in the measured water content. Although, lacking an applicable reference method, quantitative assessment of absolute errors of both the local displacement and the local water content analyses is not feasible, the overall confidence level of the results is reflected by the deformation and wetting test cases, the results of which are shown in Figs. 5 and 7.

In order to minimize total attenuation of the X-ray beam, plastic materials were used in the sample holder. Furthermore, the thickness of the sample holder tube was relatively low. The high swelling stress generated by the sample was thereby able to induce small elastic strain in the sample holder both in radial and axial directions. Consequently, the volume and the shape of the sample do not remain exactly constant during the experiments, as indicated by the measured displacement vectors near the sample surfaces. This, however, is not detrimental for the intended use of the data in validating models for water transport and solid phase deformation, as the location of the sample boundaries at each instant of time can also be estimated from the tomographic images.

Some qualitative observations concerning the inherently quite complicated process of wetting and swelling of bentonite in a confined conditions can already be made based on the measured data visualized in Figs. 8 through 11. Firstly, in the present set-up where no external water pressure was used, wetting does not seem to take place as a well defined wetting front that would be characteristic to e.g. simple capillary action. Instead, the observed water transport mechanism resembles diffusion which, however, may not be of a simple linear type. Secondly, in the initial phase of wetting, the material is first pushed away from the wetted zone, apparently due to rapid swelling near the water entrance region. At intermediate times however, the direction of displacements can be inverted in parts of the sample volume such that the total movement can be towards the water entrance region. As the material approaches its final saturated state, the displacement field again becomes more homogeneous 
and unidirectional. A plausible explanation for this rather complicated behavior is provided by collapse (leading to decrease of porosity) of the material at intermediate values of water content. This phenomenon is also indicated by the axial swelling stress measured at the nonwetting end of the sample (see Fig. 11), showing a typical behavior of stress build-up with a temporary decrease of stress after initial rapid increase followed by final increase towards saturation value (Nishimura et al., 2012). However, in view of the X-ray tomographic results shown in Fig. 9, it is obvious that interpretation of the measured evolution of the axial stress in terms of material swelling properties is not quite straightforward. The mean axial stress measured at one end of the sample is affected by combined effects of inhomogeneous local wetting and stress-strain history, and most likely also by frictional stress between the sample and the sample holder surfaces. More thorough analysis of the results obtained by the experimental methods developed here will be presented elsewhere.

\section{Conclusions}

A method for simultaneous non-intrusive analysis of three-dimensional deformation and water transport in solid, wetting material, based on X-ray tomographic imaging has been introduced. The material is assumed to consist of three components: solid, water and gas that are homogeneously distributed in the scale of imaging resolution. The attenuation of X-rays in the gas phase is neglected. The analysis is based on comparing the tomographic images of the reference state and of a wetted and deformed state. The displacement field is obtained by a straightforward image correlation method. This requires that a sufficient amount of local details, identifiable in the two images, are found in both states, and that the imaging resolution is sufficient for revealing the deformations. The deformation analysis was successfully compared with numerical solution for a rubber test sample under axial compression. The analysis of water content is based on the local grayscale value differences between the reference state and the wetted state. It utilizes the measured deformation field to compute changes of the solid phase partial density from the reference state. The method requires calibration with samples with known solid and water content. The water content analysis was compared with gravimetric results from axially wetted and sliced cylindrical bentonite samples. The results showed relatively good accuracy in the interior parts of the sample, but considerable error near both end boundaries of the sample. A plausible source of this error is the conical X-ray beam geometry and the rather simple standard algorithm used for tomographic image reconstruction.

The methods were applied here in a 4D study (three spatial dimensions and time) of wetting and swelling deformation of a purified sodium bentonite doped with small glass tracer particles, and wetted with a synthetic groundwater of salinity $0.26 \mathrm{~g} / \mathrm{l}$. The results appear qualitatively correct and plausible, and provide valuable information for e.g. validation of models involving transport of water and the resulting swelling deformation of bentonite. Further analysis of the experimental results and comparison with model predictions are left for a future work.

While the primary motivation and field of application in this work have been the study of the hydromechanical properties and swelling of bentonite, the developed method is potentially applicable in also other materials and processes involving liquid transport and deformation. 
This may include wetting/swelling and drying/shrinking of various materials provided that they contain sufficient amount of local inhomogeneities to allow for displacement analysis, and that the presence of water in the material is observable by X-ray imaging technique.

\section{Acknowledgments}

This work was funded by the Finnish Research Programme on Nuclear Waste Management KYT2014 through the project "Assessment of bentonite characteristics (BOA)". The authors appreciate the contribution by Pirkko Hölttä (University of Helsinki, Laboratory of Radiochemistry) who provided the synthetic groundwater used in the wetting experiments.

\section{References}

Anderson, S. H., Gantzer, C. J., Boone, J. M., and Tully, R. J. (1988). Rapid nondestructive bulk density and soil-water content determination by computed tomography. Soil Science Society of America Journal, 52(1):35-40.

Aregawi, W., Defraeye, T., Saneinejad, S., Vontobel, P., Lehmann, E., Carmeliet, J., Derome, D., Verboven, P., and Nicolai, B. (2013). Dehydration of apple tissue: intercomparison of neutron tomography with numerical modelling. International Journal of Heat and Mass Transfer, 67:173-182.

Barrett, J. F. and Keat, N. (2004). Artifacts in CT: recognition and avoidance. Radiographics, 24(6):1679-1691.

Bart-Smith, H., Bastawros, A.-F., Mumm, D. R., Evans, A. G., Sypeck, D. J., and Wadley, H. N. G. (1998). Compressive deformation and yielding mechanisms in cellular Al alloys determined using X-ray tomography and surface strain mapping. Acta Materialia, 46(10):3583-3592.

Carmeliet, J. and Roels, S. (2001). Determination of the isothermal moisture transport properties of porous building materials. Journal of Building Physics, 24(3):183-210.

Gardner, C. M. K., Robinson, D., Blyth, K., and Cooper, J. D. (2000). Soil water content. In Soil and Environmental Analysis: Physical Methods, pages 1-64. CRC Press.

Herrmann, K.-H., Pohlmeier, A., Gembris, D., and Vereecken, H. (2002). Three-dimensional imaging of pore water diffusion and motion in porous media by nuclear magnetic resonance imaging. Journal of Hydrology, 267(3):244-257.

Hsieh, J., Nett, B., Yu, Z., Sauer, K., Thibault, J.-B., and Bouman, C. A. (2013). Recent advances in CT image reconstruction. Current Radiology Reports, 1(1):39-51.

Hubbell, J. H. and Seltzer, S. M. (1996). Tables of X-ray Mass Attenuation Coefficients and Mass Energy-Absorption Coefficients. National Institute of Standards and Technology. 
Huisman, J. A., Hubbard, S. S., Redman, J. D., and Annan, A. P. (2003). Measuring soil water content with ground penetrating radar. Vadose zone journal, 2(4):476-491.

Huitti, T., Hakanen, M., and Lindberg, A. (1998). Sorption of Cesium on Olkiluoto Mica Gneiss, Granodiorite and Granite. Posiva Oy.

Meinzer, F. C. (2002). Co-ordination of vapour and liquid phase water transport properties in plants. Plant, Cell \& Environment, 25(2):265-274.

Moldrup, P., Olesen, T., Komatsu, T., Schjønning, P., and Rolston, D. E. (2001). Tortuosity, diffusivity, and permeability in the soil liquid and gaseous phases. Soil Science Society of America Journal, 65(3):613-623.

Mukhlisin, M., Saputra, A., El-Shafie, A., and Taha, M. R. (2012). Measurement of dynamic soil water content based on electrochemical capacitance tomography. International Journal of Electrochemical Science, 7(6).

Nishimura, T., Koseki, J., and Matsumoto, M. (2012). Measurement of swelling pressure for bentonite under relative humidity control. In Unsaturated Soils: Research and Applications, pages 235-240. Springer.

Orteu, J.-J. (2009). 3-D computer vision in experimental mechanics. Optics and Lasers in Engineering, 47(3):282-291.

Peth, S., Nellesen, J., Fischer, G., and Horn, R. (2010). Non-invasive 3D analysis of local soil deformation under mechanical and hydraulic stresses by $\mu \mathrm{CT}$ and digital image correlation. Soil and Tillage Research, 111(1):3-18.

Riedel, I., Andò, E., Salager, S., Bésuelle, P., and Viggiani, G. (2012). Water retention behaviour explored by X-ray CT analysis. In Unsaturated Soils: Research and Applications, pages 81-88. Springer.

Saguy, I. S., Marabi, A., and Wallach, R. (2005). New approach to model rehydration of dry food particulates utilizing principles of liquid transport in porous media. Trends in Food Science \& Technology, 16(11):495-506.

Sammartino, S., Michel, E., and Capowiez, Y. (2012). A novel method to visualize and characterize preferential flow in undisturbed soil cores by using multislice helical CT. Vadose Zone Journal, 11(1).

Soo, S. L. (1990). Multiphase Fluid Dynamics. Science Press, Beijing, China.

Stock, S. R. (2008). Microcomputed Tomography: Methodology and Applications. CRC press.

Tippkötter, R., Eickhorst, T., Taubner, H., Gredner, B., and Rademaker, G. (2009). Detection of soil water in macropores of undisturbed soil using microfocus X-ray tube computerized tomography $(\mu \mathrm{CT})$. Soil and Tillage Research, 105(1):12-20. 
Valton, S., Peyrin, F., and Sappey-Marinier, D. (2006). Analysis of cone-beam artifacts in offcentered circular CT for four reconstruction methods. International Journal of Biomedical Imaging, 2006.

Watanabe, S. (1996). Elastic analysis of axi-symmetric finite cylinder constrained radial displacement on the loading end. Structural Engineering/Earthquake Engineering, 13:175186.

Wildenschild, D., Hopmans, J. W., Rivers, M. L., and Kent, A. J. R. (2005). Quantitative analysis of flow processes in a sand using synchrotron-based X-ray microtomography. Vadose Zone Journal, 4(1):112-126.

Zou, W., Hunter, N., and Swain, M. V. (2011). Application of polychromatic $\mu$ CT for mineral density determination. Journal of dental research, 90(1):18-30. 